\title{
Motor Function in the Late Phase After Stroke: Stroke Survivors' Perspective
}

\author{
Lina Bunketorp-Kåll, $\mathrm{PhD}^{1,2}$, Marcela Pekna, MD, PhD ${ }^{1,3,4}$, Milos Pekny, MD, PhD ${ }^{1,3,4}$, \\ Hans Samuelsson, $\mathrm{PhD}^{1,5}$, Christian Blomstrand, $\mathrm{MD}, \mathrm{PhD}^{1,6}$, Michael Nilsson, $\mathrm{MD}, \mathrm{PhD}^{1,3,7,8,9}$ \\ ${ }^{1}$ Center for Brain Repair, Department of Clinical Neuroscience, Institute of Neuroscience and Physiology, \\ University of Gothenburg, Gothenburg, Sweden; \\ ${ }^{2}$ Center for Advanced Reconstruction of Extremities (CARE), Sahlgrenska University Hospital, Mölndal, Sweden; \\ ${ }^{3}$ Florey Institute of Neuroscience and Mental Health, Melbourne, Australia; \\ ${ }^{4}$ University of Newcastle, Newcastle, Australia; \\ ${ }^{5}$ Department of Psychology, University of Gothenburg, Gothenburg, Sweden; \\ ${ }^{6}$ Stroke Center West, Department of Clinical Neuroscience, Institute of Neuroscience and Physiology, The Sahlgrenska Academy \\ at the University of Gothenburg, Gothenburg, Sweden; \\ ${ }^{7}$ Centre for Rehab Innovations (CRI), University of Newcastle, Newcastle, Australia; \\ ${ }^{8}$ Hunter Medical Research Institute, Newcastle, Australia; \\ ${ }^{9}$ Lee Kong Chian School of Medicine, Nanyang Technological University, Singapore, Singapore
}

\begin{abstract}
Objective To examine the association between observer-assessed functional status and perceived recovery in the late phase after stroke. The study also aimed to determine whether observer-assessed functional improvements as a result of horse-riding therapy (H-RT) are related to enhanced perception of stroke recovery.

Methods This is a descriptive correlational study using data derived from a three-armed randomized controlled trial in which 123 individuals were enrolled, among whom 43 received H-RT for 12 weeks. The measures included the Modified Motor Assessment Scale, Berg Balance Scale, Timed Up and Go, timed 10-m walk, and perceived recovery from stroke indicated by item \#9 in the Stroke Impact Scale (version 2.0). Spearman rank order correlation $\left(r_{s}\right)$ was used in the analyses.

Results There were moderate to strong positive or negative correlations between all four observer-assessed motor variables and participants' ratings of perceived late-phase stroke recovery at trial entrance, ranging from $r_{s}=-0.49$ to $r_{s}=0.54$ ( $p<0.001$ ). The results of the correlational analyses of variable changes showed that, after the end of the H-RT intervention, both selfselected and fast gait speed improvement were significantly correlated with increments in self-rated stroke recovery $\left(r_{s}=-0.41\right.$, $\mathrm{p}=0.01$ and $\mathrm{r}_{\mathrm{s}}=-0.38, \mathrm{p}=0.02$, respectively).

Conclusion This study provided data supporting the association between individual ratings of self-perceived recovery after stroke and observer-assessed individual motor function. The results further demonstrate that enhancement in perceived stroke recovery after completing the intervention was associated with objectively measured gains in both self-selected and fast gait speed.
\end{abstract}

Keywords Stroke, Recovery, Horseback riding therapies, Walking speed, Motor activity

Received March 25, 2020; Revised April 30, 2020; Accepted June 3, 2020; Published online September 28, 2020

Corresponding author: Lina Bunketorp-Käll

Center for Advanced Reconstruction of Extremities (CARE), Sahlgrenska University Hospital/Mölndal, House U1, 6 th floor, 43180 Mölndal, Sweden. Tel: +46-709-723101, Fax:+46-31-873009, E-mail: lina.bunketorp-kall@neuro.gu.se

ORCID: Lina Bunketorp-Käll (https://orcid.org/0000-0002-4571-0335); Marcela Pekna (https://orcid.org/0000-0003-2734-8237); Milos Pekny (https:// orcid.org/0000-0003-1607-8075); Hans Samuelsson (https://orcid.org/0000-0003-3753-8317); Christian Blomstrand (https://orcid.org/0000-00033503-860X); Michael Nilsson (https://orcid.org/0000-0002-8826-1621).

(c) This is an open-access article distributed under the terms of the Creative Commons Attribution Non-Commercial License (http://creativecommons.org/ licenses/by-nc/4.0) which permits unrestricted noncommercial use, distribution, and reproduction in any medium, provided the original work is properly cited. Copyright (C) 2020 by Korean Academy of Rehabilitation Medicine 


\section{INTRODUCTION}

Stroke is a heterogeneous condition with complex symptomatology, and despite recent advances in treatment and rehabilitation, the condition often leads to persistent disability [1]. Many stroke survivors have motor impairment [2]. Balance deficits, spasticity, and dyscoordinated gait due to hemiparesis dispose stroke survivors to sedentary behaviors, which hamper postural control ability and increase the risk for falls [3-5]. Impaired mobility may also lead to significant activity limitations and participation restriction $[1,6]$, thereby increasing social isolation and loneliness [7-9]. Even though rehabilitation programs for patients with stroke include gait training, many individuals still have motor impairment after completion of standard rehabilitation. Improved walking ability is a prioritized goal among stroke survivors, and recovery of impaired motor functionsis a major aspect of stroke rehabilitation [2].

We have previously found that horse-riding therapy ( $\mathrm{H}$ RT) and rhythm- and music-based therapy (R-MT) helps promote subjective and objective functional recovery in the late phase after stroke $[10,11]$, including gains in motor function. Additional in-depth analyses of data derived from the randomized controlled trial (RCT) supported the observed efficacy of $\mathrm{H}$-RT in producing immediate and sustained gains in gait and functional mobility in the late phase after stroke, whereas the effectiveness of R-MT in this respect was less convincing [12].

Evaluation of individual recovery following stroke is critical for both treatment and research purposes [13]. Functional assessments reveal that certain aspects of individual dysfunction are related to the degree of recovery, such as neurological deficit (e.g., hemiparesis or aphasia), performance of specific tasks (e.g., feeding oneself or walking), function in normal roles and activities (e.g., employment or hobbies), and quality of life [14]. Patientreported outcome measures (PROMs) are useful in addition to observer-assessed measures in assessing health status of individuals with stroke [15]. In some cases, PROMs may even be better than observer-assessed measures in identifying changes in health status of relevance for the patient [16]. However, whether the degree of observer-assessed motor recovery in the late phase after stroke is associated with the degree of perceived stroke recovery is unclear.
Previous studies have demonstrated that gait speed is a reliable, valid, and sensitive measure of recovery after stroke that reflects impaired mobility and functional community walking ability [17-19]. Gait speed is also a common indicator used to predict future health status and healthcare utilization among older adults [20,21]. It has been demonstrated that improvements in walking speed are correlated with improved participation and quality of life $[18,22]$. Moreover, improvement in walking speed may reflect a genuine improvement in mobility, even if other measures fail to detect it [23]. This study aimed to assess the extent to which subjective ratings of perceived recovery after stroke are associated with observer-assessed functional status. It also aimed to determine whether observer-assessed functional improvements resulting from H-RT could be linked to enhanced perception of stroke recovery.

\section{MATERIALS AND METHODS}

\section{Study design and participants}

This is a descriptive correlational study using data derived from a three-armed RCT that assessed whether R-MT and H-RT could lead to increased recovery in the late phase after stroke $[10,11]$. The RCT enrolled 123 participants, the eligibility criteria were limited to individuals who had stroke between 10 months and 5 years prior to inclusion in the trial who had disability/dependence equivalent to grades 2 and 3 on the modified Rankin Scale (mRS).

The observer-assessed measurements of motor function and gait were conducted by a trained physiotherapist, and a subset of the data collected at baseline was used to study the association between functional status and perceived recovery after stroke $(n=123)$. Data describing the change in observer-assessed functional measures at intervention completion and 6-month follow-up was used to determine whether an increase in perceived stroke recovery was associated with gains in functional measures in the H-RT group ( $\mathrm{n}=43$ ). All participants provided written informed consent. Consenting individuals attended an appointment with a specialist in rehabilitation medicine for a neurological screening assessment and interview prior to inclusion. Ethical approval was granted by the Regional Ethical Review Board in Gothenburg (No. 698-09), and the study was conducted in accor- 
dance with relevant ethical guidelines.

\section{Measures}

\section{Stroke severity and disability}

To objectively quantify stroke-related neurologic deficits, the National Institutes of Health Stroke Scale (NIHSS) was used. The NIHSS was originally designed as a research tool to measure baseline data inpatients of acute stroke clinical trials but is now also widely used as a clinical routine assessment tool. The NIHSS has been shown to be reliable and predictive of both short- and long-term outcome following stroke $[24,25]$.

\section{Perceived stroke recovery}

Prior to randomization in the RCT, the global perception of recovery from stroke was evaluated using item \#9 in the Stroke Impact Scale (SIS, version 2.0). The item was presented in the form of a visual analogue scale, and participants were asked to rate their recovery on a scale of 0 to 100, with 100 representing full recovery. The SIS can be used in both clinical and research settings and is shown to be valid, reliable, and sensitive to change [26].

\section{Observer-assessed balance function}

Balance ability was evaluated using Berg Balance Scale. The test contains 14 elements, adding up to a total score (maximum 56). A score $<45$ may indicate an increased risk of falling and therefore the need of assistance. The test has high validity, reliability, and inter-and intra-rater reliability [27].

\section{Observer-assessed mobility and gait}

Basic functionalmobility was assessed using the Timed Up and Go (TUG). The time taken to complete the TUG was measured in seconds using a stopwatch. The TUG is a well-documented test of functional balance with high validity and reliability [28].

Gait speed was assessed using the timed 10 -m walk test [29]. The test was performed in a straight corridor from a standing start with the participant positioned behind a starting line marked with tape on the floor. The timer was started once the subject initiated walking and their first foot passed the starting line. The timer was stopped when the subject's first foot passed the end of the $10-\mathrm{m}$ pathway marked with tape. Walking time was measured in seconds for both self-perceived comfortable pace and fast pace. Walking aids were allowed, but the same aids had to be used on all test occasions. No physical assistance was permitted. The test is considered to have high interrater and test-retest reliability and low variability $[30,31]$.

\section{Observer-assessed functional mobility performance}

Daily motor function was assessed using the Modified Motor Assessment Scale according to Uppsala University Hospital (M-MAS UAS) [32]. The scale was developed based on Carr and Shepherd's theories of motor relearning after stroke [33]. The scale was based on performance and assessed performance of functional tasks rather than isolated patterns of movement. It has been modified since 1991 and validated in different studies. In this study, the M-MAS UAS version 1999 was used. The MMAS UAS was used to assess eight motor components in individuals with stroke: supine to side lying, supine to sitting over side of bed, sitting, sitting to standing, walking, upper arm function, hand movements, and fine motor activities. The latter three components were assessed bilaterally. Each item was scored from 1 to 5; the maximum score of 55 indicates optimal motor function. Motor control was measured in eight levels. To provide a global perspective of patients' functional mobility capacity, the total M-MAS score was used.

\section{Statistical analysis}

Descriptive statistics were used for demographic characteristics of the sample. Categorical variables were presented as number and percentage. Continuous variables were described using mean and standard deviation (SD). To explore the association between observer-assessed functional statusand participants' perception of stroke recovery at baseline in the RCT, Spearman rank-order correlation was used. The strength of the relationships was classified as low $(\mathrm{r}<0.30)$, moderate $(0.30 \leq \mathrm{r} \leq 0.50)$, and high ( $r>0.50)$ [34]. Spearman rank-order correlation was also used in investigating whether observer-assessed functional improvements following $\mathrm{H}-\mathrm{RT}$ are related to increments in self-rated stroke recovery after treatment. Analyses were performed using the Statistical Package for the Social Sciences (SPSS) version 24.0 (IBM Corp., Armonk, NY, USA). All statistical tests were two-tailed, and a p-value less than 0.05 was considered statistically significant. 


\section{Intervention}

The H-RT was performed at a riding center purposebuilt for the disabled where trained therapy horses were used. Participants attended twice weekly sessions of $\mathrm{H}$-RT during a 12-week intervention period. The H-RT was designed to stimulate motor, sensory, cognitive, emotional, and social functions. A description of the $\mathrm{H}$-RT content and expected therapeutic benefits has been previously presented in detail $[10,11]$.

\section{RESULTS}

The study cohort of 123 individuals (44\% women and $56 \%$ men) had a mean age of 63 years $(S D=6.5)$. The mean elapsed time since stroke onset for the study group was 1.156 days $(\mathrm{SD}=484)$. Demographics and clinical characteristics are presented in Table 1. The NIHSS scores indicated mild to moderate stroke severity, with a mean score of $2.77(\mathrm{SD}=2.81)$. Table 2 presents the Spearman correlation coefficients describing the association between the observer-assessed functional measures and

Table 1. Demographic and clinical characteristics of study participants $(\mathrm{n}=123)$

\begin{tabular}{lc}
\hline \multicolumn{1}{c}{ Variable } & Value \\
\hline Sex & \\
\hline Female & $54(44)$ \\
Male & $69(56)$ \\
\hline Age (yr) & $62.7 \pm 6.7$ \\
Time since stroke onset (day) & $1.156 \pm 484$ \\
Modified Rankin Scale & \\
\hline Grade 2 & $72(56)$ \\
\hline Grade 3 & $51(42)$ \\
SIS stroke recovery & $59.3 \pm 19.9$ \\
\hline Median (min-max) & $60(0-98)$ \\
M-MAS UAS (0-55) & $50.6 \pm 6.2$ \\
BBS (0-56) & $52.0 \pm 5.8$ \\
TUG (s) & $11.5 \pm 5.1$ \\
\hline Timed 10mWT (s) & $11.22 \pm 4.9$ \\
\hline Self-selected & $8.21 \pm 4.0$ \\
\hline Fast
\end{tabular}

Values are presented as number (\%) or mean \pm standard deviation.

M-MAS UAS, Modified Motor Assessment Scale according to Uppsala University Hospital version 99; SIS, Stroke Impact Scale; TUG, Timed Up and Go; 10mWT, 10-meter walk test. perceived stroke recovery at baseline. There were moderate to strong correlations between all motor variables and perception of stroke recovery, among which the performance of daily motor tasks (M-MAS) was most strongly related to perceived recovery $\left(\mathrm{r}_{\mathrm{s}}=0.54 ; \mathrm{p}<0.001\right)$.

Demographics and clinical characteristics of the participants who were enrolled in the H-RT group are presented in Table 3. The NIHSS scores of the H-RT group

Table 2. Spearman correlation coefficients describing the association between observer-assessed motor function and perceived stroke recovery at baseline of the intervention study

\begin{tabular}{lcc}
\hline \multicolumn{1}{c}{ Variable } & Stroke recovery $\left(\mathbf{r}_{\mathbf{s}}\right)$ & p-value \\
\hline M-MAS UAS & 0.54 & $<0.001$ \\
BBS & 0.51 & $<0.001$ \\
TUG & -0.51 & $<0.001$ \\
Timed 10mWT & & \\
Self-selected & -0.49 & $<0.001$ \\
Fast & -0.49 & $<0.001$ \\
\hline
\end{tabular}

M-MAS UAS, Modified Motor Assessment Scale according to Uppsala University Hospital version 99; BBS, Berg Balance Scale; TUG, Timed Up and Go; 10mWT, 10-meter walk test.

Table 3. Demographic and clinical characteristics of horse-riding therapy participants $(n=43)$

\begin{tabular}{|c|c|}
\hline Variable & Value \\
\hline \multicolumn{2}{|l|}{ Sex } \\
\hline Female & $16(41)$ \\
\hline Male & $23(59)$ \\
\hline Age (yr) & $62.7 \pm 6.5$ \\
\hline \multicolumn{2}{|c|}{ Modified Rankin Scale } \\
\hline Grade 2 & $23(59)$ \\
\hline Grade 3 & $16(41)$ \\
\hline M-MAS UAS & $50.33 \pm 6.1$ \\
\hline BBS $(0-56)$ & $50.4 \pm 5.7$ \\
\hline TUG (s) & $14.3 \pm 8.0$ \\
\hline \multicolumn{2}{|l|}{ Timed 10mWT (s) } \\
\hline Self-selected & $12.7 \pm 6.1$ \\
\hline Fast & $9.2 \pm 4.8$ \\
\hline
\end{tabular}

Values are presented as number (\%) or mean \pm standard deviation.

M-MAS UAS, Modified Motor Assessment Scale according to Uppsala University Hospital version 99; BBS, Berg Balance Scale; TUG, Timed Up and Go; 10mWT, 10-meter walk test. 
Table 4. Spearman correlation coefficients describing the association between therapy-induced gains in motor function and perceived increments in stroke recovery after receiving horse-riding therapy

\begin{tabular}{lcccc}
\hline \multicolumn{1}{c}{ Variable } & Stroke recovery at post-intervention $\left(\mathbf{r}_{\mathbf{s}}\right)$ & $\mathbf{p}$-value & Stroke recovery at $\mathbf{6}$ months $\left(\mathbf{r}_{\mathbf{s}}\right)$ & p-value \\
\hline M-MAS UAS & 0.13 & 0.40 & 0.21 & 0.18 \\
BBS & 0.25 & 0.12 & 0.19 & 0.25 \\
TUG & -0.04 & 0.81 & -0.09 & 0.56 \\
Timed 10mWT & & & & 0.20 \\
Self-selected & -0.41 & 0.01 & -0.21 & 0.15 \\
Fast & -0.38 & 0.02 & -0.24 & \\
\hline
\end{tabular}

M-MAS UAS, Modified Motor Assessment Scale according to Uppsala University Hospital version 99; BBS, Berg Balance Scale; TUG, Timed Up and Go; 10mWT, 10-meter walk test.
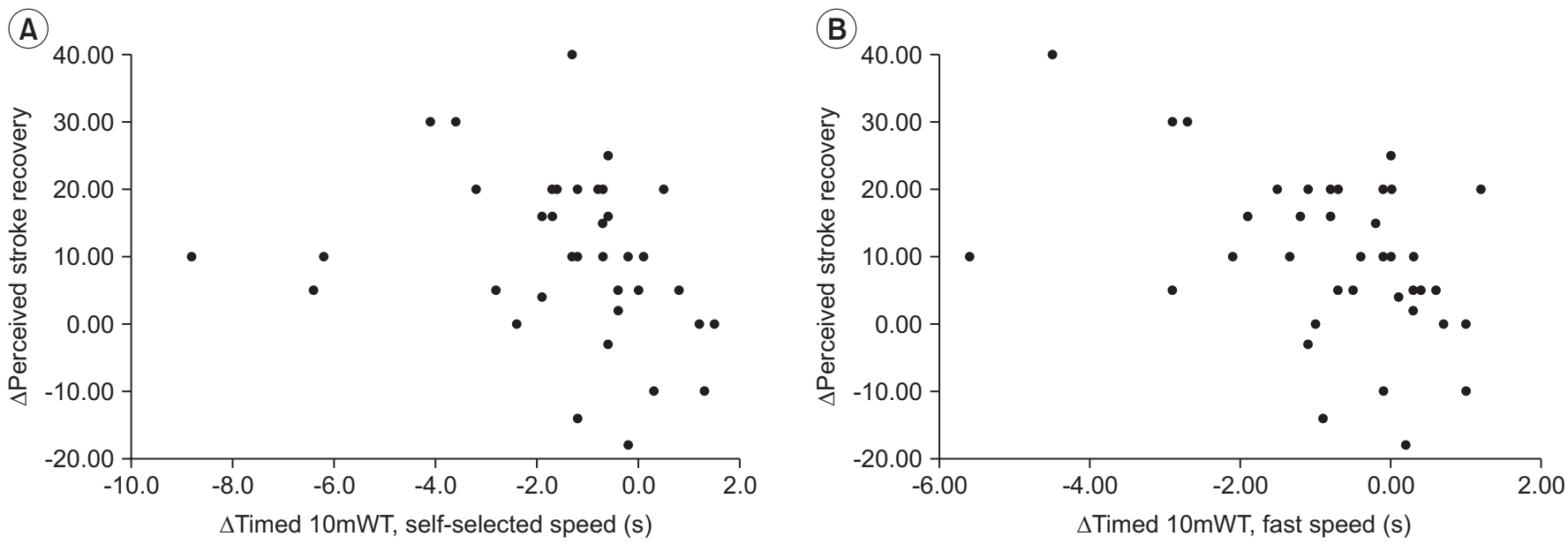

Fig. 1. Scatter plots for the association between gains in self-selected gait speed (A) and fast gait speed (B), and increments in perceived stroke recovery after end of horse-riding therapy.

indicated mild to moderate stroke severity, with a mean score of 2.62 ( $\mathrm{SD}=3.18)$. The correlational analyses of thevariable changes showed that, at post-intervention, both self-selected and fast gait speed improvement was significantly correlated with increments in self-rated stroke recovery (Table 4). In Fig. 1, scatter plots for the association between gains in gait speed and increments in perceived stroke recovery at the end of treatment are shown. The correlations between changes in these variables were no longer significant at the 6-month follow-up.

\section{DISCUSSION}

The findings of the present study demonstrated that the degree of observer-assessed motor function among latephase stroke survivors enrolled in a clinical study could be related to subjective ratings of perceived recovery since the stroke onset. It is of interest that a subjective global measure of recovery is associated with the degree of persistent motor deficitsin the late phase after stroke. Daily motor function measured using M-MAS UAS was linked to the perception of recovery, as was balance and gait ability. Hence, the ability to perform functional tasks and isolated patterns of movement or postural control functions, such as balance and gait, seem to be related to perceived disability among stroke survivors.

Furthermore, data in the present study also provided the opportunity to assess the longitudinal relationship between perceived stroke recovery and observerassessed functional improvements after an intervention. These complementary analyses showed that changes in the individual's perception of recovery after completing the 12-week H-RT intervention was associated with objectively measured gains in both self-selected and fast gait speed. Participants with greater gait-speed improvement reported greater perceived recovery compared to 
participants with less gait speed gains after the H-RT. This finding highlights the importance of using interventions designed to improve walking ability, not only in the acute and subacute phases after stroke but also in later stages of the disease. Improved gait ability has been described as one of the most important goals in individuals with stroke undergoing rehabilitation [35] and those living with stroke in the community [35]. The ability to voluntarily increase gait speed from slow to fast speed helps stroke survivors to adapt better in activities of community life, such as crossing a street and facing unpredictable pedestrian and street traffic [36].

The potential limitations of this study should be considered when interpreting the results. One of these limitations are the eligibility criteria in the RCT, restricting our study population to individuals with moderate disability after stroke (mRS grades 2 and 3). This limits extrapolation of our findings to a population of stroke survivors with either very mild (mRS 1) or more severe disability (mRS 4). In contrast, the study enrolled a mixed population of late-phase stroke survivors who had completed conventional rehabilitation programs and were living at home with chronic stroke-related disability.

Therefore, this study demonstrates an association between individual ratings of self-perceived stroke recovery and observer-assessed individual motor function in the late phase after stroke onset.The results further demonstrate that enhancement in perceived recovery among late-phase stroke survivors after completing a 12-week $\mathrm{H}-\mathrm{RT}$ intervention was associated with objectively measured gains in both self-selected and fast gait speed.

\section{CONFLICT OF INTEREST}

No potential conflict of interest relevant to this article was reported.

\section{ACKNOWLEDGMENTS}

This work was supported by grants from Sten A Olsson Foundation for Research and Culture, the Swedish Brain Foundation, the Swedish Arts Council, the Swedish state under the agreement between the Swedish government and county councils, the ALF agreement (No. ALFGBG-716591, ALFGBG-146051), AFA Insurance, the Swedish Stroke Association, Rune and Ulla Amlöv's Founda- tion for Neurological and Rheumatological Research, Edith Jacobson Foundation, Per-Olof Ahl Foundation for Neurological Research, Sigurd and Elsa Goljes Memorial Foundation, Wilhelm and Martina Lundgren Scientific Foundation, Doktor Felix Neubergh's Foundation, the Swedish Society of Medicine, and the Foundation for Rehabilitation and Medical Science.

\section{AUTHOR CONTRIBUTION}

Conceptualization: Bunketorp-Käll L, Samuelsson H. Formal analysis: Bunketorp-Käll L. Writing - original draft: Bunketorp-Käll L. Writing - review and editing: Pekna M, Pekny M, Samuelsson H, Blomstrand C, Nilsson M. Approval of final manuscript: all authors.

\section{REFERENCES}

1. Langhorne P, Bernhardt J, Kwakkel G. Stroke rehabilitation. Lancet 2011;377:1693-702.

2. Langhorne P, Coupar F, Pollock A. Motor recovery after stroke: a systematic review. Lancet Neurol 2009;8:74154.

3. Ikai T, Kamikubo T, Takehara I, Nishi M, Miyano S. Dynamic postural control in patients with hemiparesis. Am J Phys Med Rehabil 2003;82:463-9.

4. Weerdesteyn V, de Niet M, van Duijnhoven HJ, Geurts AC. Falls in individuals with stroke. J Rehabil Res Dev 2008;45:1195-213.

5. Sunnerhagen KS, Olver J, Francisco GE. Assessing and treating functional impairment in poststroke spasticity. Neurology. 2013 Jan 15;80(3 Suppl 2):S35-44.

6. Andrenelli E, Ippoliti E, Coccia M, Millevolte M, Cicconi B, Latini L, et al. Features and predictors of activity limitations and participation restriction 2 years after intensive rehabilitation following first-ever stroke. Eur J Phys Rehabil Med 2015;51:575-85.

7. Venna VR, Xu Y, Doran SJ, Patrizz A, McCullough LD. Social interaction plays a critical role in neurogenesis and recovery after stroke. Transl Psychiatry 2014;4:e351.

8. Alzahrani M, Dean C, Ada L. Relationship between walking performance and types of community-based activities in people with stroke: an observational study. Rev Bras Fisioter 2011;15:45-51.

9. Rand D, Eng JJ, Tang PF, Jeng JS, Hung C. How active 
are people with stroke?: use of accelerometers to assess physical activity. Stroke 2009;40:163-8.

10. Bunketorp-Kall L, Lundgren-Nilsson A, Samuelsson H, Pekny T, Blomve K, Pekna M, et al. Long-term improvements after multimodal rehabilitation in late phase after stroke: a randomized controlled trial. Stroke 2017;48:1916-24.

11. Bunketorp Kall L, Lundgren-Nilsson A, Blomstrand C, Pekna M, Pekny M, Nilsson M. The effects of a rhythm and music-based therapy program and therapeutic riding in late recovery phase following stroke: a study protocol for a three-armed randomized controlled trial. BMC Neurol 2012;12:141.

12. Bunketorp-Kall L, Pekna M, Pekny M, Blomstrand C, Nilsson M. Effects of horse-riding therapy and rhythm and music-based therapy on functional mobility in late phase after stroke. NeuroRehabilitation 2019;45:483-92.

13. Lee KB, Lim SH, Kim KH, Kim KJ, Kim YR, Chang WN, et al. Six-month functional recovery of stroke patients: a multi-time-point study. Int J Rehabil Res 2015;38:173-80.

14. Kasner SE. Clinical interpretation and use of stroke scales. Lancet Neurol 2006;5:603-12.

15. Groeneveld IF, Goossens PH, van Meijeren-Pont W, Arwert HJ, Meesters JJL, Rambaran Mishre AD, et al. Value-based stroke rehabilitation: feasibility and results of patient-reported outcome measures in the first year after stroke. J Stroke Cerebrovasc Dis 2019;28:499-512.

16. Katzan IL, Thompson NR, Lapin B, Uchino K. Added value of patient-reported outcome measures in stroke clinical practice. J Am Heart Assoc 2017;6:e005356.

17. Lee KB, Kim JH, Lee KS. The relationship between motor recovery and gait velocity during dual tasks in patients with chronic stroke. J Phys Ther Sci 2015;27:1173-6.

18. Schmid A, Duncan PW, Studenski S, Lai SM, Richards L, Perera S, et al. Improvements in speed-based gait classifications are meaningful. Stroke 2007;38:2096100.

19. Patterson SL, Forrester LW, Rodgers MM, Ryan AS, Ivey FM, Sorkin JD, et al. Determinants of walking function after stroke: differences by deficit severity. Arch Phys Med Rehabil 2007;88:115-9.

20. Montero-Odasso M, Schapira M, Soriano ER, Va- rela M, Kaplan R, Camera LA, et al. Gait velocity as a single predictor of adverse events in healthy seniors aged 75 years and older. J Gerontol A Biol Sci Med Sci 2005;60:1304-9.

21. Sartor-Glittenberg C, Lehmann S, Okada M, Rosen D, Brewer K, Bay RC. Variables explaining health-related quality of life in community-dwelling older adults. J Geriatr Phys Ther 2014;37:83-91.

22. Lord SE, McPherson K, McNaughton HK, Rochester L, Weatherall M. Community ambulation after stroke: how important and obtainable is it and what measures appear predictive? Arch Phys Med Rehabil 2004;85:234-9.

23. Kollen B, Kwakkel G, Lindeman E. Hemiplegic gait after stroke: is measurement of maximum speed required? Arch Phys Med Rehabil 2006;87:358-63.

24. Goldstein LB, Samsa GP. Reliability of the National Institutes of Health Stroke Scale: extension to nonneurologists in the context of a clinical trial. Stroke 1997;28:307-10.

25. Appelros P, Terent A. Characteristics of the National Institute of Health Stroke Scale: results from a population-based stroke cohort at baseline and after one year. Cerebrovasc Dis 2004;17:21-7.

26. Duncan PW, Wallace D, Lai SM, Johnson D, Embretson S, Laster LJ. The stroke impact scale version 2.0. Evaluation of reliability, validity, and sensitivity to change. Stroke 1999;30:2131-40.

27. Berg K, Wood-Dauphinee S, Williams JI. The Balance Scale: reliability assessment with elderly residents and patients with an acute stroke. Scand J Rehabil Med 1995;27:27-36.

28. Ng SS, Hui-Chan CW. The timed up \& go test: its reliability and association with lower-limb impairments and locomotor capacities in people with chronic stroke. Arch Phys Med Rehabil 2005;86:1641-7.

29. Duncan PW, Sullivan KJ, Behrman AL, Azen SP, Wu SS, Nadeau SE, et al. Protocol for the Locomotor Experience Applied Post-stroke (LEAPS) trial: a randomized controlled trial. BMC Neurol 2007;7:39.

30. Tyson S, Connell L. The psychometric properties and clinical utility of measures of walking and mobility in neurological conditions: a systematic review. Clin Rehabil 2009;23:1018-33.

31. Perry J, Garrett M, Gronley JK, Mulroy SJ. Classification of walking handicap in the stroke population. 
Stroke 1995;26:982-9.

32. Andersson C, Clevnert M. Reliability testing of the Modified Motor Assessment Scale according to Uppsala University Hospital (M-MAS UAS-99). Uppsala, Sweden: Uppsala University; 2000.

33. Carr JH, Shepherd RB, Nordholm L, Lynne D. Investigation of a new motor assessment scale for stroke patients. Phys Ther 1985;65:175-80.
34. Cohen J. Statistical power analysis for the behavioral sciences. 2nd ed. Hillsdale, NJ: Erlbaum; 1988.

35. Harris JE, Eng JJ. Goal priorities identified through client-centred measurement in individuals with chronic stroke. Physiother Can 2004;56:171-6.

36. Ng SS, Ng PC, Lee CY, Ng ES, Tong MH. Walkway lengths for measuring walking speed in stroke rehabilitation. J Rehabil Med 2012;44:43-6. 